\title{
Adaptive Beamforming with Side Lobe Suppression by Placing Extra Radiation Pattern Nulls
}

\author{
Ioannis P. Gravas, Student Member, IEEE, Zaharias D. Zaharis, Senior Member, IEEE, Traianos V. \\ Yioultsis, Member, IEEE, Pavlos I. Lazaridis, Senior Member, IEEE, and Thomas D. Xenos
}

\begin{abstract}
A new iterative adaptive beamforming (ABF) algorithm based on conventional beamformers is proposed, in order not only to steer the main lobe towards a desired signal and place radiation pattern nulls towards respective interference signals, but also to achieve a desired side lobe level (SLL). Thus, the algorithm becomes less susceptible to unpredicted interference signals than conventional beamformers. In each iteration, the algorithm finds the direction of the peak of the greatest side lobe, which is considered as direction of arrival (DoA) of a hypothetical interference signal, and the conventional beamformer is then employed to find proper antenna array weights that produce an extra null towards this direction. The iterative procedure stops when the desired SLL is obtained. The algorithm is applied on three conventional beamformers and is tested for various signal DoA, while the direction deviation of the main lobe and the nulls is recorded, to evaluate the algorithm in terms of robustness. The proposed algorithm needs a few iterations to achieve the desired SLL, and thus is much faster than any evolutionary iterative method employed for side lobe suppression. Finally, unlike methods that employ neural networks, the proposed algorithm does not need any training to become functional.
\end{abstract}

Index Terms - Adaptive beamforming, minimum variance distortionless response, recursive least squares, sample matrix inversion, side lobe level, side lobe suppression.

\section{INTRODUCTION}

A DAPTIVE beamforming ( $\mathrm{ABF}$ ) is a real-time procedure applied on the feeding of antenna array elements [1]-[24]. The term "adaptive" indicates the ability of this procedure to dynamically steer the antenna array main lobe at the direction of arrival (DoA) of a desired incoming signal (DIS) and place a null at the DoA of every respective undesired incoming signal (UIS). Since the DoA of every signal is time varying, the $\mathrm{ABF}$ technique has to find at every moment the appropriate weights of the array elements, that create a main lobe towards the DoA of DIS and place nulls towards the respective DoA of UISs. The final goal is to maximize the signal to interference-plus-noise ratio (SINR). Since sample sets of DIS and UISs are used in the beamforming procedure,
ABF belongs into the category of digital beamforming, which yields better results compared to conventional analog beamforming [25].

Several deterministic [2]-[5], [7], [8], [10], [12], [15], [17], [20], [23] and evolutionary ABF techniques [6], [9], [11], [13], [14], [16], [18], [19], [21], [22], [24] can be found in the literature, as well as applications of beamforming in different types of antennas and radars [23], [24], [26]-[34]. Among these techniques, the minimum variance distortionless response (MVDR), sample matrix inversion (SMI), and recursive least squares (RLS) [2], [3] are very popular. Like every conventional ABF technique, MVDR, SMI and RLS do not take into account the side lobe level (SLL) and therefore they cannot minimize it. On the other hand, new interference signals from various angles of arrival (AoA) may arise during the time span that a certain data sample set is processed by the beamformer to estimate the correlation matrices. Thus, the radiation pattern nulls created at the end of this time span cannot take into account these new interference signals. Such signals with DoA close to directions of strong side lobes lead to SINR degradation. Also, by ignoring the SLL, some radiated power is being wasted, resulting in poor power efficiency.

The proposed algorithm has iterative structure and aims in each iteration at finding the direction of the strongest side lobe in order to guide the ABF technique (e.g., MVDR, SMI or RLS) to consider this direction as DoA of a hypothetical UIS. Thus, in each iteration, the ABF technique is employed to find proper weights that steer the main lobe towards the DoA of DIS and concurrently place nulls towards the respective DoA of the actual UISs together with additional nulls towards the respective DoA of all the hypothetical UISs found at the end of the current iteration and the previous ones. In this way, every strong side lobe found until the end of the current iteration splits into two smaller lobes. It is expected that these additional nulls help to suppress all the strong side lobes found during the iterative process. This process continues until SLL $\leq-20 \mathrm{~dB}$ with respect to the main lobe peak, which is considered enough to suppress unexpected interference signals. To the best of the authors' knowledge, extra radiation pattern nulls placed at the directions of strong side lobes have never been used so far by beamforming techniques to reduce the SLL. The proposed algorithm is computationally light and fast, due to the small number of iterations needed to achieve the desired SLL.

For the purpose of this study, the proposed algorithm is applied to a linear array of thirty ideal omni-directional sources $(M=30)$ uniformly spaced at distance $d$ equal to $\lambda / 2$ 
$(d=\lambda / 2)$, where $\lambda$ is the free space wavelength. Since the elements are ideal, there is no coupling between them, and the radiation pattern is calculated only by the antenna array factor. One DIS and two UISs are assumed to be concurrently received by the antenna array in the presence of zero-mean additive Gaussian noise with a certain signal to noise ratio (SNR). The MVDR, SMI and RLS are alternatively employed here by the proposed algorithm to estimate the antenna array weights that achieve proper main lobe steering together with null placing at desired directions. Also, we apply time average estimation of the correlation matrices in MVDR and SMI by using blocks of data samples. This makes the results become more realistic.

The novelty introduced in this paper is the incorporation of well-known $\mathrm{ABF}$ techniques into a properly structured iterative algorithm to achieve not only main lobe and null control but also SLL control, which is not provided by conventional ABF techniques. So, several advantages are achieved by the proposed algorithm: Firstly, the computational procedure is light and is completed in a few iterations, making thus the algorithm capable of responding immediately in real time. Therefore, the algorithm is much faster than any evolutionary iterative method employed for side lobe suppression, since an evolutionary method usually needs several hundreds of iterations to come to a conclusion. Secondly, the SLL reduction is beneficial, because it helps the beamformer to suppress any interference signal, which has not been taken into account during the processing time span. Thirdly, unlike methods that employ neural networks (NNs), the proposed algorithm does not need any training to become functional. Finally, the algorithm seems to work efficiently, even when AoA of UISs are very close to AoA of DIS, as shown in the statistical results presented in section VIII.

\section{PRIOR ART}

Several techniques can be found in the literature that aim at reducing the SLL. In [13], particle swarm optimization is used to achieve harmonic beam steering with low SLL by defining on-off sequences of excitations in time-modulated linear arrays. In [14], ABF is achieved by applying a variant of an evolutionary method called invasive weed optimization. ABF techniques based on NNs are proposed in [16] and [19] in order to control the SLL. A genetic algorithm using Pareto front selection is introduced in [18] in order to provide beam patterns with reduced SLL in random antenna arrays. In [20], SLL reduction is achieved by decreasing the peak SLL of the Capon spectrum. An iterative algorithm called numerical pattern synthesis (NPS) is proposed in [35]. According to this algorithm, a very large number (two or three times the degrees of freedom of the antenna array) of artificial interference signals is used with their power being updated at each iteration until the desired SLL is achieved. In [23], [24] and [36] convex optimization is applied for beamforming and side lobe suppression. A low side lobe pattern synthesis method is proposed in [37] for large planar arrays. The method makes use of successive Fast Furrier Transforms (FFTs) of the array factor. Several methods of array pattern synthesis with low SLL can also be found in [38]. In [39], an iterative fast Fourier technique is introduced for null control. This technique needs
2000 iterations to achieve the required null depth and the desired SLL, while only broadside cases were studied. A beamforming network based on Nolen matrix topology for use in low SLL linear multibeam antennas is introduced in [40]. In [41], SLL reduction and null control are applied to linear arrays by using Taguchi's method. In this study, only broadside cases are considered, while the excitation weights are assumed to have symmetrically distributed amplitudes with respect to the center of the array and no phase differences. Nevertheless, the number of iterations determines the accuracy of the optimized solution derived by Taguchi's method. Therefore, the number of iterations must be increased when an increased accuracy is required, or when the number of elements that compose the array increases, or when nonbroadside cases are studied (i.e., the excitation weights are composed of non-symmetrical amplitudes and unequal phases). In [42], a side lobe suppression algorithm is applied to holographic metamaterial antennas. The algorithm relies on the values of two parameters, which are optimized by applying backtracking search and the gradient descend method. In [43], the NPS algorithm is employed in conjunction with a determination procedure of the main lobe region, in order to synthesize 2-D patterns with low SLL. In [44], a low side lobe level time-domain adaptive beamforming technique has been proposed using convex optimization. A diagonal loading technique is introduced in [45] to keep the SLL at a desired value using Taylor excited antennas. Concentric ring isophoric sparse arrays are proposed in [46] to achieve optimal pattern shaping. Such arrays can effectively be used to perform static beamforming (for invariable environments) and concurrently achieve low SLL. In [47], a new method based on discrete dipole approximation is introduced to design metasurface antennas with low SLL. A novel pattern synthesis method is proposed in [48] to obtain low SLL. The method is applied to unequally spaced linear arrays, takes into account the mutual coupling between the array elements and is implemented by using iterative FFTs via virtual active element pattern expansion. Finally, in [49], thinned fractal antenna arrays are used to achieve main lobe steering together with null control and SLL reduction. The SLL reduction and the control of a single null are concurrently achieved by applying a combination of ant colony optimization (which is a discrete evolutionary method) and a least mean squares algorithm.

A general comment would be that all the side lobe suppression techniques that employ evolutionary optimization methods encounter a great difficulty in responding promptly in practice, due to the large number of iterations required by the evolutionary methods. It is obvious that an iterative method can be used for real time applications only if a few iterations are required by the method to produce a result, as is the case of our proposed algorithm. Finally, it has to be noted that side lobe suppression techniques that employ NNs exhibit instant response, but the NNs must undergo a procedure of proper training as well as an evaluation procedure in order to become functional.

\section{FORMULATION}

According to the conventional narrowband beamforming problem, $N+1$ monochromatic signals at a wavelength $\lambda$ are 
received by a linear array composed of $M$ ideal omnidirectional sources uniformly spaced at distance $d=\lambda / 2$, while it is assumed that $M>N$ [2]. The array is assumed to be parallel to the z-axis. In general, the signals concurrently received by the antenna array are a DIS $s_{0}$ with AoA defined as $\theta_{0}$ and $N$ UISs $s_{n}(n=1, \ldots, N)$ with respective AoA defined as $\theta_{n}(n=1, \ldots, N)$, as shown in Fig. 1. In this study, it is considered that $N=2$ and $M=30$. Each AoA is defined by the DoA of the respective signal and the direction which is normal to the axis of the antenna array (see Fig. 1). Actually, each AoA is complementary to the elevation angle defined here by the DoA of the respective signal and z-axis.

The mean power of DIS is defined as

$$
P_{S 0}=E\left[s_{0}(k) s_{0}^{*}(k)\right],
$$

where $\mathrm{E}[\cdot]$ denotes the expected value and index $k$ indicates the $k$-th time sample. $P_{S 0}$ is considered as reference power for all signals and is therefore assumed to be equal to one. The signal $x_{m}(k)$ created at the input of the $m$-th array element $(m=1, \ldots, M)$ includes a zero-mean additive Gaussian noise signal $n_{m}(k)$. Since $n_{1}(k), \ldots, n_{M}(k)$ are zero-mean signals, their variance $\sigma^{2}$ will be equal to their mean power which, in turn, can be calculated by the value of the SNR.

If we use blocks of $K$ time samples $(k=1, \ldots, K)$, the input signals $x_{m}(m=1, \ldots, M)$ can be expressed by an $M \times K$ input signal matrix given as follows:

$$
\mathbf{X}(b)=\mathbf{a}_{0} \mathbf{s}_{0}(b)+\mathbf{A} \mathbf{S}(b)+\mathbf{N}(b),
$$

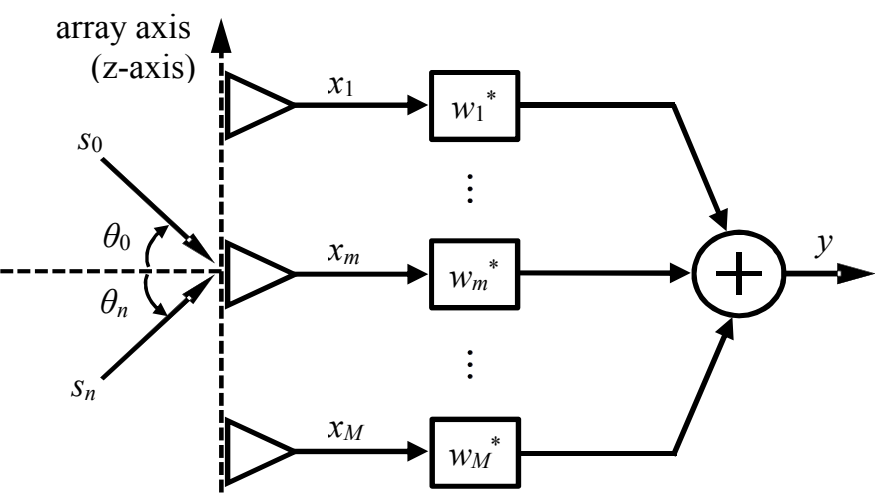

Fig. 1. Adaptive beamformer block diagram.

where $b$ is the sequence number of the sample block. To clarify all the vectors and matrices in (2), we give the following definitions:

$$
\mathbf{a}_{n}=\left[\begin{array}{llll}
1 & e^{j \beta d \sin \theta_{n}} & \cdots & e^{j(M-1) \beta d \sin \theta_{n}}
\end{array}\right]^{T}, n=0,1, \ldots, N
$$

and

$$
\mathbf{A}=\left[\begin{array}{lll}
\mathbf{a}_{1} & \cdots & \mathbf{a}_{N}
\end{array}\right]
$$

are, respectively, the array steering vector that corresponds to a certain AoA $\theta_{\mathrm{n}}$, and the array steering matrix constructed by the steering vectors that correspond to AoA of all UISs $(n=1, \ldots, N)$. Also in (3), $\beta$ is the free space wavenumber $(\beta=2 \pi / \lambda)$, and superscript $T$ indicates the transpose operation.
In addition,

$$
\begin{aligned}
& \mathbf{s}_{n}(b)=\left[\begin{array}{lll}
s_{n}(1+(b-1) K) & \cdots & s_{n}(K+(b-1) K)
\end{array}\right], \\
& n=0,1, \ldots, N
\end{aligned}
$$

and

$$
\mathbf{S}(b)=\left[\begin{array}{lll}
\mathbf{s}_{1}(b) & \cdots & \mathbf{s}_{N}(b)
\end{array}\right]^{T}
$$

are, respectively, a row vector that contains the $K$ samples of the $b$-th sample block of incoming signal $s_{n}$, and the UIS sample matrix constructed by the row vectors that correspond to all UISs. Also,

$$
\begin{aligned}
& \mathbf{n}_{m}(b)=\left[\begin{array}{lll}
n_{m}(1+(b-1) K) & \cdots & n_{m}(K+(b-1) K)
\end{array}\right], \\
& m=1, \ldots, M
\end{aligned}
$$

and

$$
\mathbf{N}(b)=\left[\begin{array}{lll}
\mathbf{n}_{1}(b) & \cdots & \mathbf{n}_{M}(b)
\end{array}\right]^{T}
$$

are, respectively, a row vector that contains the $K$ samples of the $b$-th sample block of noise signal $n_{m}$, and the noise sample matrix constructed by the noise row vectors that correspond to all array elements. As shown in (2), $\mathbf{X}$ is the sum of three terms. The first term is due to DIS, the second is due to UISs, and finally the third is due to noise signals. Therefore, equation (2) can be written in the form

$$
\mathbf{X}(b)=\mathbf{X}_{d}(b)+\mathbf{X}_{u}(b)=\mathbf{X}_{d}(b)+\mathbf{X}_{i}(b)+\mathbf{N}(b),
$$

where

$$
\begin{gathered}
\mathbf{X}_{d}(b)=\mathbf{a}_{0} \mathbf{s}_{0}(b), \\
\mathbf{X}_{i}(b)=\mathbf{A S}(b),
\end{gathered}
$$

and

$$
\mathbf{X}_{u}(b)=\mathbf{X}_{i}(b)+\mathbf{N}(b)=\mathbf{A} \mathbf{S}(b)+\mathbf{N}(b)
$$

are, respectively, the desired, the interference and the total undesired component of $\mathbf{X}$.

For each signal, a respective correlation matrix is defined. We assume that the beamforming process is ergodic in correlation. Thus, a statistical correlation matrix can be well approximated by the respective time average estimate of this matrix constructed from time samples of the corresponding signal. The correlation matrix of $x_{m}$ is defined as

$$
\mathbf{R}_{x x}(b)=\frac{1}{K} \mathbf{X}(b) \mathbf{X}^{H}(b),
$$

where superscript $H$ indicates the Hermitian transpose operation. Similar definitions can be given for the correlation matrices that correspond to $x_{d m}$ (desired component of $x_{m}$ ), $x_{i m}$ (interference component of $x_{m}$ ) and $n_{m}$. By considering that there is no correlation among $x_{d m}, x_{i m}$ and $n_{m}$, we easily get

$$
\mathbf{R}_{x x}(b)=\mathbf{R}_{d d}(b)+\mathbf{R}_{i i}(b)+\mathbf{R}_{n n}(b)=\mathbf{R}_{d d}(b)+\mathbf{R}_{u u}(b)
$$

where $\mathbf{R}_{u u}$ is the correlation matrix of the total undesired 
signal component (i.e., $x_{u m}=x_{i m}+n_{m}, m=1, \ldots, M$ ).

The beamformer output $y$ can be expressed by the following row vector:

$$
\mathbf{y}(b)=\mathbf{w}^{H}(b) \mathbf{X}(b)
$$

where

$$
\mathbf{w}(b)=\left[\begin{array}{lll}
w_{1}(b) & \cdots & w_{M}(b)
\end{array}\right]^{T}
$$

is the excitation weight vector that corresponds to the $b$-th sample block. From (9) and (15), it is obvious that the output can be considered as the sum of a desired and an undesired component:

$$
\begin{aligned}
\mathbf{y}(b) & =\mathbf{y}_{d}(b)+\mathbf{y}_{u}(b)=\mathbf{w}^{H}(b) \mathbf{X}_{d}(b)+\mathbf{w}^{H}(b) \mathbf{X}_{u}(b) \\
& =\mathbf{w}^{H}(b) \mathbf{a}_{0} \mathbf{s}_{0}(b)+\mathbf{w}^{H}(b)[\mathbf{A S}(b)+\mathbf{N}(b)] .
\end{aligned}
$$

Since the mean power of DIS was assumed to be equal to one, the mean power of $y_{d}$ is given as

$$
P_{y d}(b)=E\left[\mathbf{y}_{d}(b) \mathbf{y}_{d}^{H}(b)\right]=\mathbf{w}^{H}(b) \mathbf{a}_{0} \mathbf{a}_{0}^{H} \mathbf{w}(b) .
$$

On the other hand, the mean power of $y_{u}$ is given as

$$
\begin{aligned}
& P_{y u}(b)=E\left[\mathbf{y}_{u}(b) \mathbf{y}_{u}^{H}(b)\right]= \\
& =\mathbf{w}^{H}(b) \mathbf{A} \mathbf{R}_{s s}(b) \mathbf{A}^{H} \mathbf{w}(b)+\mathbf{w}^{H}(b) \mathbf{R}_{n n}(b) \mathbf{w}(b),
\end{aligned}
$$

where $\mathbf{R}_{s s}$ is the correlation matrix of UISs $(n=1, \ldots, N)$. This matrix is approximated by a time average estimate as

$$
\mathbf{R}_{s s}(b)=\frac{1}{K} \mathbf{S}(b) \mathbf{S}^{H}(b),
$$

where $\mathbf{S}(b)$ is given by (6). Equation (19) is extracted provided that there is no correlation between any two incoming signals and no correlation between any incoming signal and noise. Finally, (18) and (19) can be used to extract the value of the SINR that corresponds to the $b$-th sample block:

$$
\begin{aligned}
& \operatorname{SINR}(b)=P_{y d}(b) / P_{y u}(b)= \\
& =\frac{\mathbf{w}^{H}(b) \mathbf{a}_{0} \mathbf{a}_{0}^{H} \mathbf{w}(b)}{\mathbf{w}^{H}(b) \mathbf{A} \mathbf{R}_{s s}(b) \mathbf{A}^{H} \mathbf{w}(b)+\mathbf{w}^{H}(b) \mathbf{R}_{n n}(b) \mathbf{w}(b)} .
\end{aligned}
$$

\section{Minimum VARIANCE Distortionless RESPONSE}

The MVDR technique aims at finding the optimum weight vector $\mathbf{w}$ that minimizes $P_{y u}$, while the desired output signal $y_{d}$ is not distorted, resulting thus in maximizing the SINR [2]. It must be noted that the MVDR solution is identical to the Maximum Likelihood (ML) solution, with the only difference that the ML approach needs all UISs and noise signals to have zero mean value and follow Gaussian distribution. Thus, the MVDR solution is more general. The optimum $\mathbf{w}$ is given by the following expression:

$$
\mathbf{w}_{M V D R}(b)=\frac{\mathbf{R}_{x x}^{-1}(b) \mathbf{a}_{0}}{\mathbf{a}_{0}^{H} \mathbf{R}_{x x}^{-1}(b) \mathbf{a}_{0}} .
$$

\section{SAMPLE MATRIX INVERSION}

In the SMI technique, the optimum weights are estimated by minimizing the Mean Square Error (MSE) between the output $y$ of the beamformer and a reference signal $r$, which usually is a form of DIS [2]. The error signal vector is a row vector defined as

$$
\boldsymbol{\varepsilon}(b)=\mathbf{r}(b)-\mathbf{w}^{H}(b) \mathbf{X}(b),
$$

where

$$
\mathbf{r}(b)=\left[\begin{array}{lll}
r(1+(b-1) K) & \cdots & r(K+(b-1) K)
\end{array}\right]
$$

is also a row vector, which contains the $K$ samples of the $b$-th sample block of the reference signal. The MSE between $y$ and $r$ for the $b$-th sample block is defined as

$$
\varepsilon_{M S}(b)=\frac{1}{K} \boldsymbol{\varepsilon}(b) \boldsymbol{\varepsilon}^{H}(b)
$$

Then, by differentiating $\varepsilon_{M S}(b)$ with respect to $\mathbf{w}(b)$, and by demanding the derivative to be equal to zero, we can find the optimum weight vector that minimizes $\varepsilon_{M S}(b)$ as given below:

$$
\mathbf{w}_{S M I}(b)=\mathbf{R}_{x x}^{-1}(b) \mathbf{z}(b)
$$

where

$$
\mathbf{z}(b)=\frac{1}{K} \mathbf{X}(b) \mathbf{r}^{H}(b)
$$

is the estimate of the correlation vector between the reference signal and the array input signals for the $b$-th sample block.

\section{RECURSIVE LEAST SQUARES}

Similar to SMI, RLS is another technique that aims at minimizing the MSE. Unlike MVDR and SMI, which make use of sample blocks to estimate $\mathbf{R}_{x x}$ and then invert $\mathbf{R}_{x x}$ to calculate the array weights, the RLS technique updates directly $\mathbf{R}_{x x}^{-1}$ every time an input signal sample is acquired, reducing thus the complexity of the whole process. Nevertheless, a drawback of this technique is that it needs several iterations to converge. The RLS technique makes use of a parameter $a$ called "forgetting factor" in order to limit the effect of older samples. The update of $\mathbf{R}_{x x}^{-1}$ is achieved according to the following expression:

$$
\begin{aligned}
& \mathbf{R}_{x x}^{-1}(k)=\frac{k}{k-1}\left[a^{-1} \mathbf{R}_{x x}^{-1}(k-1)-a^{-1} \mathbf{h}(k) \mathbf{x}^{H}(k) \mathbf{R}_{x x}^{-1}(k-1)\right], \\
& k=2, \ldots, K
\end{aligned}
$$

where index $k$ indicates the $k$-th time sample, $K$ represents the total number of samples used by the technique to converge,

$$
h(k)=\frac{a^{-1} \mathbf{R}_{x x}^{-1}(k-1) \mathbf{x}(k)}{1+a^{-1} \mathbf{x}^{H}(k) \mathbf{R}_{x x}^{-1}(k-1) \mathbf{x}(k)},
$$

and finally 


$$
\mathbf{x}(k)=\left[\begin{array}{lll}
x_{1}(k) & \cdots & x_{M}(k)
\end{array}\right]^{T}
$$

is a vector that contains the $k$-th time samples of the $M$ input signals. Then, the weights are recursively updated according to the expression:

$$
\mathbf{w}_{R L S}(k)=\mathbf{w}_{R L S}(k-1)+\mathbf{h}(k)\left[r^{*}(k)-\mathbf{x}^{H}(k) \mathbf{w}_{R L S}(k-1)\right],(31
$$

where $r(k)$ is the $k$-th time sample of the reference signal. The matrix $\mathbf{R}_{x x}^{-1}$ and the weights are respectively initialized as:

$$
\mathbf{R}_{x x}^{-1}(1)=\delta \mathbf{I}_{M \times M}
$$

and

$$
\mathbf{w}_{R L S}(1)=\mathbf{0},
$$

where $\delta$ is a large arbitrary number, and $\mathbf{I}_{M \times M}$ is the $M \times M$ identity matrix. The reason that $\delta$ is chosen to be a large arbitrary number is because the RLS beamformer initially does not receive samples of the incoming signals and therefore $\mathbf{X}$ will be composed only of noise samples, which are uncorrelated and have small values. Thus, $\mathbf{R}_{x x}$ will be almost diagonal with small entries, and therefore its inverse $\left(\mathbf{R}_{x x}^{-1}\right)$ will be diagonal as well but its entries will be large. Since in the RLS beamformer, $\mathbf{R}_{x x}^{-1}$ is directly initialized (and not by inverting $\mathbf{R}_{x x}$ ), it can be approximated as an identity matrix multiplied by a large arbitrary number $\delta$. The RLS technique could also work with small values of $\delta$, but the convergence rate of the technique would be slower.

\section{Proposed Side Lobe SuPPRESSION AlgorithM}

As mentioned above, a conventional beamforming technique does not take into account the SLL. As a consequence, new interference signals may arise during the time span that a certain data sample set is processed by the beamformer. Since this sample set does not contain any information about these new interference signals, no null is created towards their DoA, resulting thus in SINR degradation especially in cases where these signals have DoA close to the directions of strong side lobes. In such cases, a low SLL would help to keep the SINR at high levels.

The proposed algorithm finds in each iteration the direction that corresponds to the strongest side lobe. Then, this direction is considered as DoA of a hypothetical UIS (interference signal), and therefore the $\mathrm{ABF}$ technique is employed by the algorithm to place a null at this direction. In this way, the strongest side lobe splits into two smaller lobes. This process is repeated until SLL $\leq-20 \mathrm{~dB}$ with respect to the main lobe peak. This means, that for a 30-element linear array $(M=30)$, proper complex values for thirty weights must be found to steer the main lobe towards DoA of DIS, place nulls towards DoA of all UISs, and also keep the SLL below $-20 \mathrm{~dB}$. Since the hypothetical UISs may actually not exist and in any case we have no information about them, a set of $K$ pseudo-samples must be generated for each hypothetical UIS, in order to make the $\mathrm{ABF}$ technique work. All pseudo-samples are random numbers following Gaussian distribution with zero mean value and variance equal to one (which is equivalent to a mean power value equal to one for each hypothetical UIS). The noise samples also follow Gaussian distribution with zero mean value and variance $\sigma^{2}$ calculated by the value of the SNR. In the case of RLS, $\mathbf{R}_{x x}^{-1}$ is reinitialized at the start of each iteration of the proposed algorithm.

It is well known that a linear array composed of $M$ ideal sources uniformly spaced at distance $d=\lambda / 2$ can produce $M-1$ nulls. In this study, we consider one DIS with AoA $\theta_{0}$ and two UISs with respective AoA $\theta_{1}$ and $\theta_{2}$. By requesting two nulls respectively at $\theta_{1}$ and $\theta_{2}$, we are left with $M-3$ available nulls (i.e., $M-3$ hypothetical UISs). Therefore, in our study, where $M=30$, the proposed algorithm must come to a conclusion at $M-3=27$ iterations at most (i.e., 27 more nulls can be placed), otherwise it is terminated regardless of whether the desired SLL has been achieved or not. The proposed algorithm is summarized in the flowchart of Fig. 2.

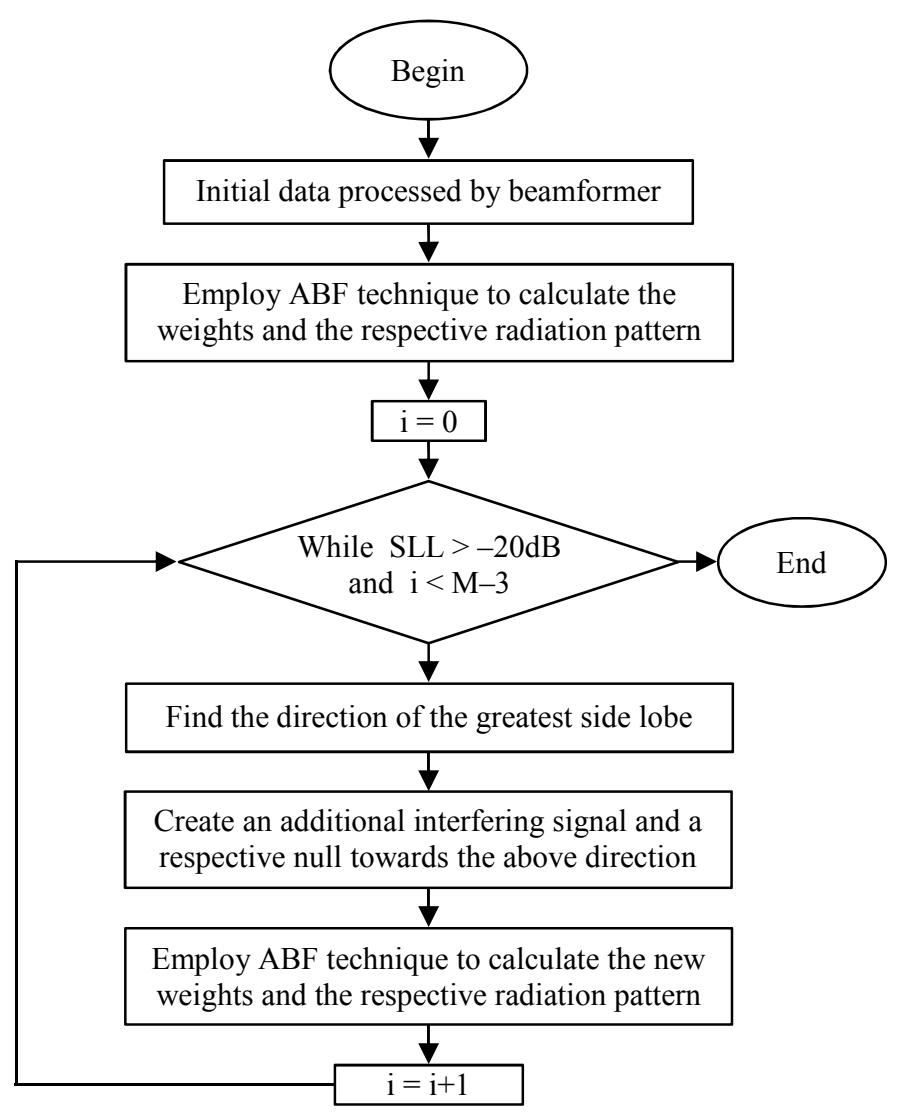

Fig. 2. Flowchart of the proposed side lobe suppression algorithm.

The application of every ABF technique always results in complex-valued weights (i.e., weights with amplitudes and phases). The proposed algorithm also results in complexvalued weights, because the algorithm employs an $\mathrm{ABF}$ technique in each iteration. Therefore, a significant problem arises concerning the feasibility of complex weights in reality. If the antenna array, utilized by the $\mathrm{ABF}$ technique or the proposed algorithm, operates in reception mode, then the implementation of complex weights and the calculation of the respective radiation pattern are easy tasks, because they are just algorithmic procedures performed by a high complexity processing unit (e.g., a multi-core CPU or GPU). Such 
procedures do not depend on the type of the elements used to compose the antenna array. However, if the antenna array operates as transmitter, the required weights must be implemented either as input currents or as input voltages (including amplitudes and phases) by using a proper active electronic circuit driven by the processing unit that has calculated these weights. The implementation of such currents or voltages is not an easy task, because it depends on the electronic circuit employed for this purpose, but is out of scope of this study. Once these currents or voltages have been implemented, it is easy to apply them to the antenna array regardless of the type of the array elements.

\section{SimULATION RESULTS}

To estimate the correlation matrices, we use blocks of either 100 or 500 samples $(K=100$ or 500$)$, for all the $\mathrm{ABF}$ techniques employed here (i.e., MVDR, SMI and RLS). To thoroughly evaluate the performance of the proposed algorithm, we run the algorithm for each possible value of $\theta_{0}$ from $-60^{\circ}$ to $+60^{\circ}$ (i.e., within a $120^{\circ}$ sector) with a step of $1^{\circ}$, while the values of $\theta_{1}$ and $\theta_{2}$ may either be both larger or both smaller or one larger and the other smaller than $\theta_{0}$. In addition, the distance $\Delta \theta$ between any adjacent two of $\theta_{0}, \theta_{1}$ and $\theta_{2}$ is kept equal to $6^{\circ}, 8^{\circ}$ or $10^{\circ}$ (i.e., three different scenarios). For every scenario, the SNR is considered to be equal to $5 \mathrm{~dB}$. It is obvious that cases with $\Delta \theta=6^{\circ}$ are the most difficult ones, because we demand either a main lobe peak between two nulls and very close to them, or a null between and very close to a main lobe peak and another null. Then, we calculate the mean value and standard deviation of the SLL, before and after the application of the proposed algorithm, as well as the mean value and standard deviation of the total number of iterations made by the algorithm to achieve the desired SLL. The results are shown in Tables I and II for 100 and 500 samples, respectively.

TABLE I

Mean Value and Standard DeViation of The SLl (Before and After The Application of The Proposed Algorithm), and Mean Value and STANDARD DEVIATION OF THE NUMBER OF ITERATIONS OF THE Proposed ALGORITHM, FOR 100 SAMPLES PER BLOCK AND SNR $=5 \mathrm{~dB}$

\begin{tabular}{cccc}
\hline & $\begin{array}{c}\text { SLL }(\mathrm{dB}) \\
\text { before } \\
\text { [mean/std] }\end{array}$ & $\begin{array}{c}\text { SLL }(\mathrm{dB}) \\
\text { after } \\
{[\mathrm{mean} / \mathrm{std}]}\end{array}$ & $\begin{array}{c}\text { Number of } \\
\text { Iterations } \\
{[\mathrm{mean} / \mathrm{std}]}\end{array}$ \\
\hline$\Delta \theta=6^{\circ}$ & & & \\
\hline MVDR & $-10.5 / 1.5$ & $-20.0 / 2.5$ & $19.0 / 6.0$ \\
SMI & $-10.5 / 1.5$ & $-19.0 / 3.5$ & $21.5 / 5.5$ \\
RLS & $-8.0 / 1.5$ & $-16.5 / 6.0$ & $26.5 / 0.5$ \\
\hline$\Delta \theta=8^{\circ}$ & & & \\
\hline MVDR & $-10.5 / 1.5$ & $-20.5 / 1.0$ & $16.5 / 4.0$ \\
SMI & $-10.0 / 1.5$ & $-20.5 / 2.0$ & $19.5 / 4.5$ \\
RLS & $-8.5 / 1.5$ & $-20.0 / 5.0$ & $26.5 / 0.5$ \\
\hline$\Delta \theta=10^{\circ}$ & & & \\
\hline MVDR & $-10.5 / 1.5$ & $-21.0 / 1.0$ & $15.0 / 3.0$ \\
SMI & $-10.0 / 1.5$ & $-21.0 / 1.0$ & $18.0 / 3.5$ \\
RLS & $-8.0 / 1.5$ & $-20.5 / 3.5$ & $26.0 / 1.0$ \\
\hline
\end{tabular}

At the same time, we calculate the average deviation of the actual main lobe direction from the respective desired direction (that corresponds to $\theta_{0}$ ) as well as the average deviation of the actual nulls directions from the respective desired directions (that corresponds to $\theta_{1}$ or $\theta_{2}$ ), before and after the application of the proposed algorithm. The results are shown in Tables III and IV for 100 and 500 samples, respectively.

TABLE II

Mean Value and Standard Deviation of The SLl (Before and After The ApPlication of The Proposed Algorithm), AND MEAN Value AND Standard DEVIaTion of THE Number of ITERATIONS OF THE Proposed

\begin{tabular}{cccc}
\multicolumn{4}{c}{ ALGORITHM, FOR 500 SAMPLES PER BLOCK AND SNR=5dB } \\
\hline & $\begin{array}{c}\text { SLL }(\mathrm{dB}) \\
\text { before } \\
{[\mathrm{mean} / \mathrm{std}]}\end{array}$ & $\begin{array}{c}\text { SLL }(\mathrm{dB}) \\
\text { after } \\
{[\mathrm{mean} / \mathrm{std}]}\end{array}$ & $\begin{array}{c}\text { Number of } \\
\text { Iterations } \\
{[\mathrm{mean} / \mathrm{std}]}\end{array}$ \\
\hline$\Delta \theta=6^{\circ}$ & & & \\
\hline MVDR & $-13.5 / 2.0$ & $-20.0 / 1.5$ & $11.5 / 8.5$ \\
SMI & $-13.5 / 2.0$ & $-20.0 / 2.0$ & $12.0 / 9.0$ \\
RLS & $-11.0 / 1.5$ & $-17.0 / 5.5$ & $26.0 / 1.0$ \\
\hline$\Delta \theta=8^{\circ}$ & & & \\
\hline MVDR & $-13.0 / 1.0$ & $-20.5 / 0.5$ & $7.0 / 4.0$ \\
SMI & $-12.5 / 1.5$ & $-20.5 / 0.5$ & $7.0 / 4.0$ \\
RLS & $-11.0 / 1.5$ & $-20.0 / 5.0$ & $26.0 / 1.0$ \\
\hline$\Delta \theta=10^{\circ}$ & & & \\
\hline MVDR & $-12.5 / 1.0$ & $-20.5 / 0.5$ & $6.0 / 1.5$ \\
SMI & $-12.5 / 1.0$ & $-20.5 / 0.5$ & $6.5 / 2.0$ \\
RLS & $-11.0 / 1.5$ & $-21.0 / 3.0$ & $25.5 / 1.5$ \\
\hline
\end{tabular}

TABLE III

Average Deviation of Main Lobe and Nulls Directions Before AND After The Application of The Proposed Algorithm, For 100 Samples

\begin{tabular}{ccccc}
\multicolumn{5}{c}{ PER BLOCK AND SNR=5dB } \\
\hline $\begin{array}{c}\text { Main lobe } \\
\text { average } \\
\text { deviation } \\
\text { before (deg) }\end{array}$ & $\begin{array}{c}\text { Main lobe } \\
\text { average } \\
\text { deviation } \\
\text { after (deg) }\end{array}$ & $\begin{array}{c}\text { Nulls } \\
\text { average } \\
\text { deviation } \\
\text { before (deg) }\end{array}$ & $\begin{array}{c}\text { Nulls } \\
\text { average } \\
\text { deviation } \\
\text { after (deg) }\end{array}$ \\
\hline$\Delta \theta=6^{\circ}$ & & & & \\
\hline MVDR & 0.20 & 0.27 & 0.06 & 0.13 \\
SMI & 0.25 & 0.37 & 0.12 & 0.20 \\
RLS & 0.29 & 0.40 & 0.13 & 0.22 \\
\hline$\Delta \theta=8^{\circ}$ & & & & \\
\hline MVDR & 0.17 & 0.25 & 0.04 & 0.10 \\
SMI & 0.19 & 0.31 & 0.10 & 0.17 \\
RLS & 0.24 & 0.34 & 0.11 & 0.18 \\
\hline$\Delta \theta=10^{\circ}$ & & & & \\
\hline MVDR & 0.15 & 0.20 & 0.03 & 0.08 \\
SMI & 0.18 & 0.23 & 0.08 & 0.15 \\
RLS & 0.23 & 0.31 & 0.10 & 0.16 \\
\hline
\end{tabular}

TABLE IV

Average Deviation of Main Lobe and Nulls Directions Before and After The Application of THe Proposed Algorithm, For 500 SAMPLES

\begin{tabular}{ccccc}
\multicolumn{5}{c}{ PER BLOCK AND SNR=5dB } \\
\hline $\begin{array}{c}\text { Main lobe } \\
\text { average } \\
\text { deviation } \\
\text { before (deg) }\end{array}$ & $\begin{array}{c}\text { Main lobe } \\
\text { average } \\
\text { deviation } \\
\text { after (deg) }\end{array}$ & $\begin{array}{c}\text { Nulls } \\
\text { average } \\
\text { deviation } \\
\text { before (deg) }\end{array}$ & $\begin{array}{c}\text { Nulls } \\
\text { average } \\
\text { deviation } \\
\text { after (deg) }\end{array}$ \\
\hline$\Delta \theta=6^{\circ}$ & & & & \\
\hline MVDR & 0.10 & 0.15 & 0.04 & 0.08 \\
SMI & 0.11 & 0.25 & 0.07 & 0.13 \\
RLS & 0.18 & 0.35 & 0.11 & 0.16 \\
\hline$\Delta \theta=8^{\circ}$ & & & & \\
\hline MVDR & 0.09 & 0.13 & 0.03 & 0.07 \\
SMI & 0.10 & 0.22 & 0.05 & 0.11 \\
RLS & 0.17 & 0.29 & 0.09 & 0.15 \\
\hline$\Delta \theta=10^{\circ}$ & & & & \\
\hline MVDR & 0.08 & 0.12 & 0.02 & 0.06 \\
SMI & 0.09 & 0.15 & 0.03 & 0.08 \\
RLS & 0.15 & 0.26 & 0.07 & 0.10 \\
\hline
\end{tabular}

As shown in Tables I and II, the proposed algorithm achieves the desired SLL in most cases. It also needs the fewest iterations to come to a conclusion when combined with the MVDR technique, and the most iterations when combined 
with the RLS technique. The standard deviations of both the SLL and the number of iterations required by the algorithm become smaller when $\Delta \theta$ increases. Also, when more samples are used (i.e., 500 instead of 100), the number of iterations required by the algorithm to converge and the standard deviation of the SLL become both smaller if the MVDR or the SMI technique is employed by the algorithm. However, the increased number of samples can neither help the algorithm finish faster nor noticeably reduce the SLL's standard deviation, when the algorithm employs the RLS technique, due to the recursive nature of this technique. It must be noted that a decrease in the SLL's standard deviation means that the algorithm achieves the desired SLL for more combinations of $\theta_{0}, \theta_{1}$ and $\theta_{2}$.

As shown in Tables III and IV, the proposed algorithm does not induce any significant difference between the main lobe direction and DoA of DIS, or between the direction of a null and DoA of the respective UIS. By using more samples, these differences become smaller, even when $\Delta \theta=6^{\circ}$ (worst case scenario).

The performance of the proposed algorithm is also evaluated in terms of SINR. For this reason, we examine six separate scenarios. The three of them use blocks of 100 samples and correspond to three different values of SNR, respectively equal to $0 \mathrm{~dB}, 5 \mathrm{~dB}$ and $10 \mathrm{~dB}$. The other three scenarios use blocks of 500 samples and correspond to the same three values of SNR as previously defined (i.e., OdB, $5 \mathrm{~dB}$ and $10 \mathrm{~dB})$. In every scenario, we run all the $\mathrm{ABF}$ techniques for each possible value of $\theta_{0}$ from $-60^{\circ}$ to $+60^{\circ}$ with a step of $1^{\circ}$, while the values of $\theta_{1}$ and $\theta_{2}$ may either be both larger or both smaller or one larger and the other smaller than $\theta_{0}$. In addition, the distance $\Delta \theta$ between any adjacent two of $\theta_{0}, \theta_{1}$ and $\theta_{2}$ is kept equal to $6^{\circ}, 8^{\circ}$ or $10^{\circ}$, defining thus three respective sub-scenarios per scenario. The mean values of SINR before and after the application of the proposed algorithm for each $\mathrm{ABF}$ technique, each sub-scenario and each scenario are displayed in Tables V and VI. It can be seen that the SINR is slightly reduced $(0.4 \mathrm{~dB}$ on average) after the application of the proposed algorithm. Nevertheless, this reduction is expected due to the slight displacement of the main lobe and the nulls after the application of the algorithm.

TABLE V

Mean Value of The SinR Before and After The ApPlication of The PROPOSED ALGORITHM, USING 100 SAMPLES PER BLOCK

\begin{tabular}{cccc}
\hline & $\begin{array}{c}\text { Mean SINR (dB) } \\
\text { for SNR=0dB } \\
\text { [before/after] }\end{array}$ & $\begin{array}{c}\text { Mean SINR (dB) } \\
\text { for SNR=5dB } \\
\text { [before/after] }\end{array}$ & $\begin{array}{c}\text { Mean SINR (dB) } \\
\text { for SNR=10dB } \\
\text { [before/after] }\end{array}$ \\
\hline$\Delta \theta=6^{\circ}$ & & & \\
\hline MVDR & $14.7 / 14.1$ & $19.7 / 19.1$ & $24.7 / 24.1$ \\
SMI & $14.4 / 13.8$ & $19.5 / 18.9$ & $24.5 / 23.9$ \\
RLS & $13.0 / 12.7$ & $18.1 / 17.7$ & $23.0 / 22.6$ \\
\hline$\Delta \theta=8^{\circ}$ & & & \\
\hline MVDR & $14.8 / 14.4$ & $19.8 / 19.3$ & $24.9 / 24.4$ \\
SMI & $14.6 / 14.0$ & $19.6 / 19.0$ & $24.6 / 24.0$ \\
RLS & $13.2 / 12.8$ & $18.2 / 17.8$ & $23.2 / 22.8$ \\
\hline$\Delta \theta=10^{\circ}$ & \multicolumn{3}{c}{} \\
\hline MVDR & $15.0 / 14.7$ & $19.9 / 19.6$ & $25.0 / 24.6$ \\
SMI & $14.7 / 14.1$ & $19.7 / 19.1$ & $24.8 / 24.2$ \\
RLS & $13.3 / 12.9$ & $18.3 / 18.0$ & $23.3 / 22.9$ \\
\hline
\end{tabular}

TABLE VI

Mean Value of The SinR Before and After The Application of The PROPOSED ALGORITHM, USING 500 SAMPLES PER BLOCK

\begin{tabular}{cccc}
\hline & $\begin{array}{c}\text { Mean SINR (dB) } \\
\text { for SNR=0dB } \\
\text { [before/after] }\end{array}$ & $\begin{array}{c}\text { Mean SINR (dB) } \\
\text { for SNR=5dB } \\
\text { [before/after] }\end{array}$ & $\begin{array}{c}\text { Mean SINR (dB) } \\
\text { for SNR=10dB } \\
\text { [before/after] }\end{array}$ \\
\hline$\Delta \theta=6^{\circ}$ & & $19.8 / 19.5$ & $24.8 / 24.3$ \\
\hline MVDR & $14.8 / 14.4$ & $19.6 / 19.0$ & $24.7 / 24.2$ \\
SMI & $14.5 / 14.0$ & $18.6 / 18.3$ & $23.6 / 23.1$ \\
RLS & $13.5 / 13.2$ & & \\
\hline$\Delta \theta=8^{\circ}$ & & $19.9 / 19.6$ & $24.9 / 24.5$ \\
\hline MVDR & $14.9 / 14.6$ & $19.8 / 19.2$ & $24.8 / 24.3$ \\
SMI & $14.8 / 14.2$ & $18.7 / 18.4$ & $23.8 / 23.3$ \\
RLS & $13.7 / 13.4$ & & \\
\hline$\Delta \theta=10^{\circ}$ & & & \\
\hline MVDR & $15.1 / 14.7$ & $20.0 / 19.7$ & $24.1 / 24.7$ \\
SMI & $15.0 / 14.4$ & $19.9 / 19.3$ & $24.0 / 23.6$ \\
RLS & $13.8 / 13.5$ & $18.8 / 18.5$ & \\
\hline
\end{tabular}

To visualize the effectiveness of the proposed algorithm, we display three figures per $\mathrm{ABF}$ technique that correspond to the three different values of $\Delta \theta$. The values of AoA for $\Delta \theta=6^{\circ}$ are $\theta_{0}=30^{\circ}, \theta_{1}=24^{\circ}$ and $\theta_{2}=36^{\circ}$, for $\Delta \theta=8^{\circ}$ are $\theta_{0}=30^{\circ}, \theta_{1}=22^{\circ}$ and $\theta_{2}=38^{\circ}$, and finally for $\Delta \theta=10^{\circ}$ are $\theta_{0}=30^{\circ}, \theta_{1}=20^{\circ}$ and $\theta_{2}=40^{\circ}$. In all cases, 500 samples are used, while the SNR is considered to be equal to $5 \mathrm{~dB}$. Figs. 3-5 display the radiation pattern before and after the application of the proposed algorithm by employing the MVDR technique for $\Delta \theta=6^{\circ}, 8^{\circ}$ and $10^{\circ}$, respectively. Figs. 6-8 display respective patterns for the SMI technique, and Figs. 9-11 display respective patterns for the RLS technique.

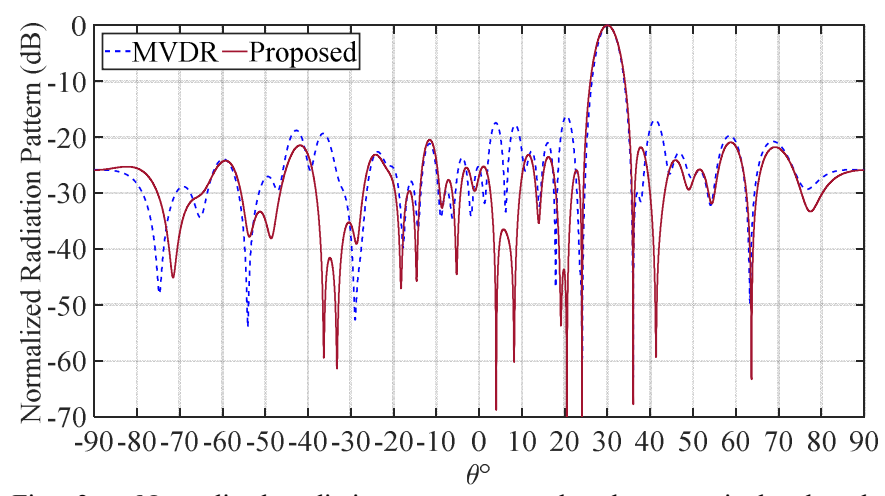

Fig. 3. Normalized radiation patterns produced, respectively, by the conventional MVDR technique, and the proposed algorithm which employs the MVDR technique, for $\theta_{0}=30^{\circ}, \theta_{1}=24^{\circ}$ and $\theta_{2}=36^{\circ}\left(\Delta \theta=6^{\circ}\right)$.

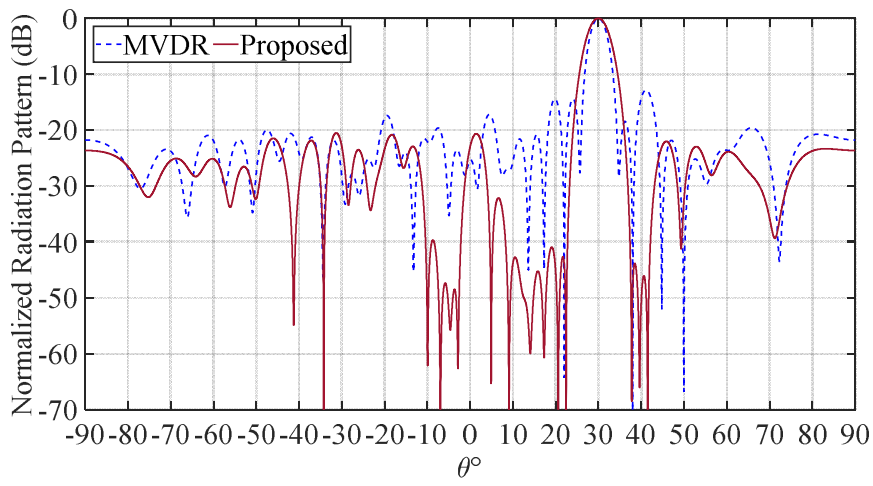

Fig. 4. Normalized radiation patterns produced, respectively, by the conventional MVDR technique, and the proposed algorithm which employs the MVDR technique, for $\theta_{0}=30^{\circ}, \theta_{1}=22^{\circ}$ and $\theta_{2}=38^{\circ}\left(\Delta \theta=8^{\circ}\right)$. 


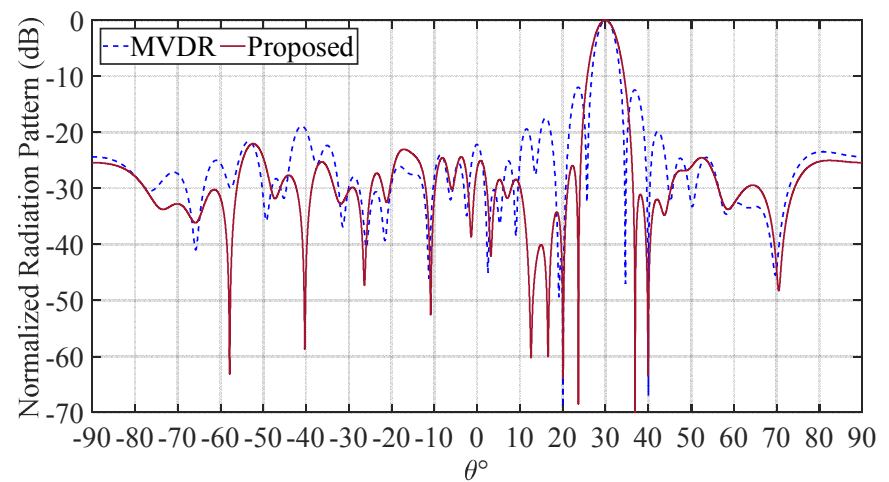

Fig. 5. Normalized radiation patterns produced, respectively, by the conventional MVDR technique, and the proposed algorithm which employs the MVDR technique, for $\theta_{0}=30^{\circ}, \theta_{1}=20^{\circ}$ and $\theta_{2}=40^{\circ}\left(\Delta \theta=10^{\circ}\right)$.

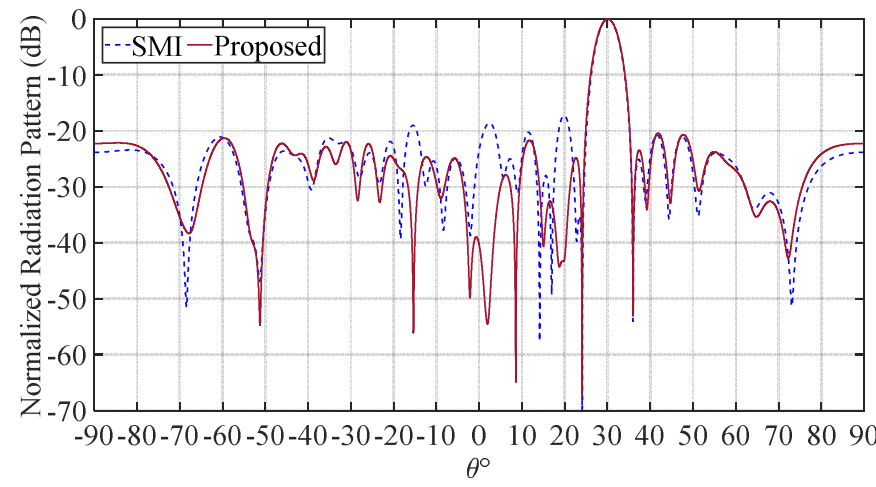

Fig. 6. Normalized radiation patterns produced, respectively, by the conventional SMI technique, and the proposed algorithm which employs the SMI technique, for $\theta_{0}=30^{\circ}, \theta_{1}=24^{\circ}$ and $\theta_{2}=36^{\circ}\left(\Delta \theta=6^{\circ}\right)$.

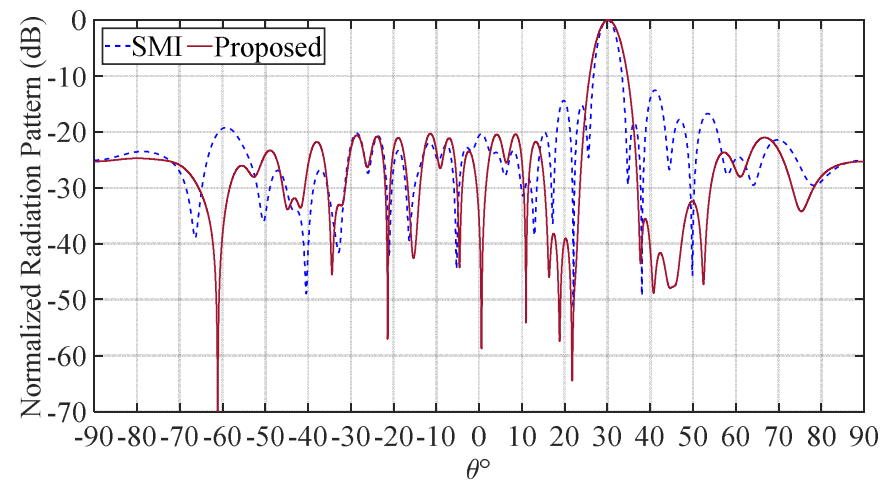

Fig. 7. Normalized radiation patterns produced, respectively, by the conventional SMI technique, and the proposed algorithm which employs the SMI technique, for $\theta_{0}=30^{\circ}, \theta_{1}=22^{\circ}$ and $\theta_{2}=38^{\circ}\left(\Delta \theta=8^{\circ}\right)$.

All the radiation patterns shown in Figs. 3-11 reveal that the SLL is reduced after the application of the proposed algorithm. It must be noted that every radiation pattern, that corresponds to a certain $\mathrm{ABF}$ technique and certain values of $\theta_{0}, \theta_{1}$ and $\theta_{2}$, may vary a bit from simulation to simulation, due to the stochastic nature of noise and interference signals. A side effect of suppressing side lobes is the slight widening of the main lobe, which is expected because a reduction in the SLL may result in power being gathered towards the main lobe, as shown in the radiation patterns. However, this does not affect the position of the nulls initially placed for the interference signals.

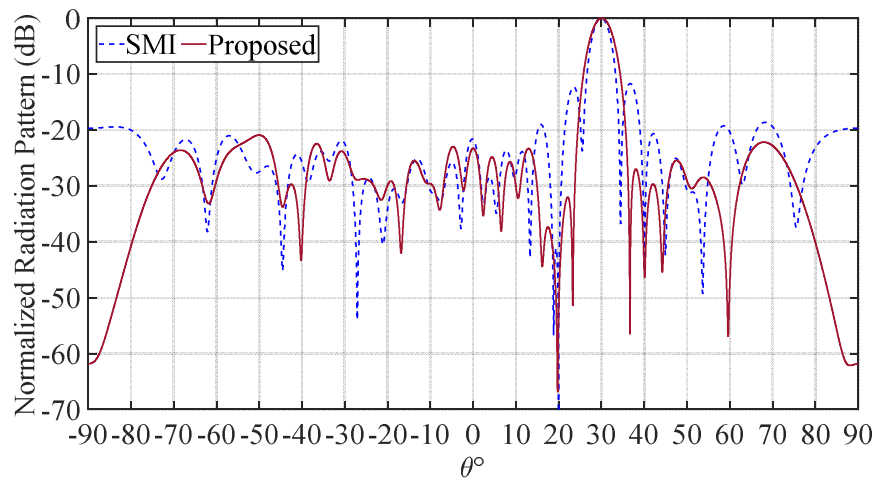

Fig. 8. Normalized radiation patterns produced, respectively, by the conventional SMI technique, and the proposed algorithm which employs the SMI technique, for $\theta_{0}=30^{\circ}, \theta_{1}=20^{\circ}$ and $\theta_{2}=40^{\circ}\left(\Delta \theta=10^{\circ}\right)$.

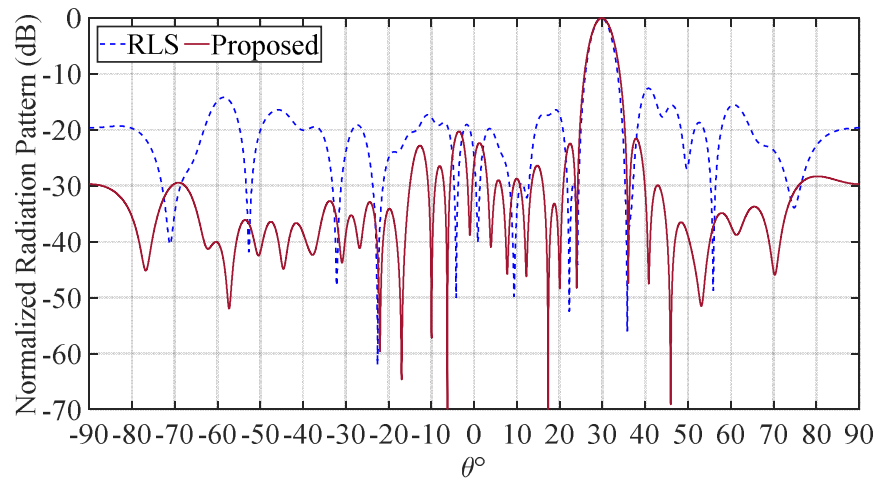

Fig. 9. Normalized radiation patterns produced, respectively, by the conventional RLS technique, and the proposed algorithm which employs the RLS technique, for $\theta_{0}=30^{\circ}, \theta_{1}=24^{\circ}$ and $\theta_{2}=36^{\circ}\left(\Delta \theta=6^{\circ}\right)$.

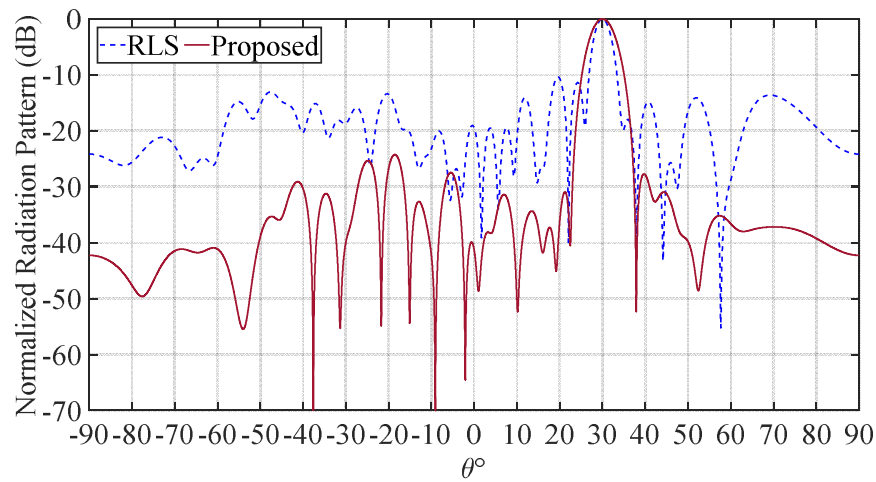

Fig. 10. Normalized radiation patterns produced, respectively, by the conventional RLS technique, and the proposed algorithm which employs the RLS technique, for $\theta_{0}=30^{\circ}, \theta_{1}=22^{\circ}$ and $\theta_{2}=38^{\circ}\left(\Delta \theta=8^{\circ}\right)$.

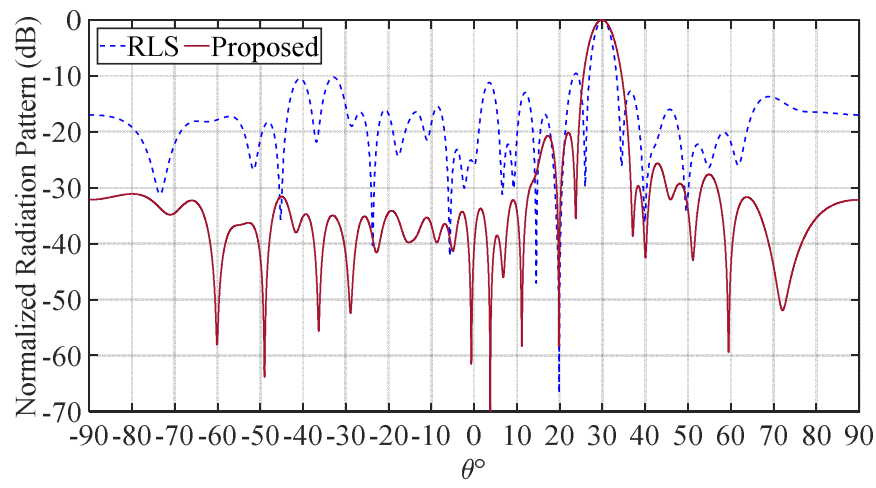

Fig. 11. Normalized radiation patterns produced, respectively, by the conventional RLS technique, and the proposed algorithm which employs the RLS technique, for $\theta_{0}=30^{\circ}, \theta_{1}=20^{\circ}$ and $\theta_{2}=40^{\circ}\left(\Delta \theta=10^{\circ}\right)$. 


\section{CONCLUSIONS}

A new $\mathrm{ABF}$ algorithm based on conventional $\mathrm{ABF}$ techniques has been introduced in order to reduce the SLL, which is not taken into account and is thus not controlled by conventional beamformers. In this way, better power efficiency is achieved, while susceptibility to unpredicted interference signals is reduced. The results derived in this study reveal that the proposed algorithm is capable of achieving its purpose after a few iterations, even when the interference signals have DoA very close to DoA of the desired signal. In addition, this algorithm does not induce any significant difference between the main lobe direction and DoA of DIS, or between the direction of a null and DoA of the respective UIS. Finally, it seems that such differences as well as the number of iterations needed by the algorithm to come to a conclusion are reduced when using more samples, improving thus the overall results even when the interference signals have DoA very close to DoA of the desired signal.

\section{ACKNOWLEDGMENTS}

This research is co-financed by Greece and the European Union (European Social Fund - ESF) through the Operational Programme "Human Resources Development, Education and Lifelong Learning" in the context of the project "Strengthening Human Resources Research Potential via Doctorate Research" (MIS-5000432), implemented by the State Scholarships Foundation (IKY).

The authors would like to thank the anonymous reviewers and the editors of the journal for their detailed and valuable comments.

\section{REFERENCES}

[1] A. H. El. Zooghby, C. G. Christodoulou, and M. Georgiopoulos, "Neural network-based adaptive beamforming for one- and two-dimensional antenna arrays," IEEE Trans. Antennas Propag., vol. 46, no. 12, pp. 1891-1893, Dec. 1998.

[2] F. B. Gross, Smart Antennas for Wireless Communications with Matlab. New York: McGraw-Hill, 2005.

[3] J. Li and P. Stoica, Robust Adaptive Beamforming. New Jersey: John Wiley \& Sons, Inc., 2006.

[4] C. C. Chai and Y. C. Liang, "Joint MIMO beamforming and power adaptation at base station and broadcasting relay transmitters with quality-of-service (QoS) constraints," in Proc. 67th IEEE Vehicular Technology Conference, Singapore, May 2008, pp. 1026-1031.

[5] Xin Song, Jinkuan Wang, and Xuefen Niu, "Robust adaptive beamforming algorithm based on neural network," in Proc. IEEE International Conference on Automation and Logistics, Qingdao, China, Sep. 2008, pp. 1844-1849.

[6] M. Roshanaei, C. Lucas, and A. R. Mehrabian, "Adaptive beamforming using a novel numerical optimisation algorithm," IET Microwaves, Antennas Propag., vol. 3, no. 5, pp. 765-773, Aug. 2009.

[7] J. A. Srar, C. Kah-Seng, and A. Mansour, "Adaptive array beamforming using a combined LMS-LMS algorithm," IEEE Trans. Antennas Propag., vol. 58, no. 11, pp. 3545-3557, Nov. 2010.

[8] J. Park and S. Lee, "MIMO beamforming for QoS enhancement via analog, digital and hybrid relaying," IEEE Trans. Broadcast., vol. 56, no. 4, pp. 494-503, Dec. 2010.

[9] Z. D. Zaharis and T. V. Yioultsis, "A novel adaptive beamforming technique applied on linear antenna arrays using adaptive mutated boolean PSO," Prog. Electromagn. Res., vol. 117, pp. 165-179, 2011.

[10] F. Liu, J. Wang, C. Y. Sun, and R. Du, "Robust MVDR beamformer for nulling level control via multi-parametric quadratic programming," Prog. Electromagn. Res. C, vol. 20, pp. 239-254, 2011.
[11] R. Mallipeddi, J. P. Lie, P. N. Suganthan, S. G. Razul, and C. M. S. See, "A differential evolution approach for robust adaptive beamforming based on joint estimation of look direction and array geometry," Prog. Electromagn. Res., vol. 119, pp. 381-394, 2011.

[12] Z. Lei, L. Wei, and R. J. Langley, "Adaptive beamforming with realvalued coefficients based on uniform linear arrays," IEEE Trans. Antennas Propag., vol. 59, no. 3, pp. 1047-1053, Mar. 2011.

[13] L. Poli, P. Rocca, G. Oliveri, and A. Massa, "Harmonic beamforming in time-modulated linear arrays," IEEE Trans. Antennas Propag., vol. 59, no. 7, pp. 2538-2545, July 2011.

[14] Z. D. Zaharis, C. Skeberis, and T. D. Xenos, "Improved antenna array adaptive beamforming with low side lobe level using a novel adaptive invasive weed optimization method," Prog. Electromagn. Res., vol. 124, pp. 137-150, 2012.

[15] M. Lertsutthiwong, T. Nguyen, and B. Hamdaoui, "Efficient wireless broadcasting through joint network coding and beamforming," Int. J. Digit. Multimed. Broadcast., vol. 2012, Article ID 342512, 2012.

[16] Z. D. Zaharis, K. A. Gotsis, and J. N. Sahalos, "Adaptive beamforming with low side lobe level using neural networks trained by mutated boolean PSO," Prog. Electromagn. Res., vol. 127, pp. 139-154, 2012.

[17] J. Park and S. Lee, "M2-m2 beamforming for virtual MIMO broadcasting in multi-hop relay networks," IEEE J. Select. Areas Commun., vol. 30, no. 8, pp. 1358-1369, Sep. 2012.

[18] S. Jayaprakasam, S. K. B. A. Rahim, and C. Y. Leow, "A Pareto elite selection genetic algorithm for random antenna array beamforming with low sidelobe level," Prog. Electromagn. Res. B, vol. 51, pp. 407-425, 2013.

[19] Z. D. Zaharis, C. Skeberis, T. D. Xenos, P. I. Lazaridis, and J. Cosmas, "Design of a novel antenna array beamformer using neural networks trained by modified adaptive dispersion invasive weed optimization based data," IEEE Trans. Broadcast., vol. 59, no. 3, pp. 455-460, Sep. 2013.

[20] K. Yang, Z. Zhao, X. Zhu, and Q. H. Liu, "Robust adaptive beamforming with low sidelobe levels," in Proc. 2013 IEEE Antennas and Propagation Society International Symposium, Orlando, FL, USA, July 2013, pp. 872-873.

[21] N. Anselmi, P. Rocca, A. Massa, and E. Giaccari, "Synthesis of robust beamforming weights in linear antenna arrays," in Proc. 2014 IEEE Conference on Antenna Measurements \& Applications, Antibes Juanles-Pins, France, Nov. 2014, pp. 1-3.

[22] N. Anselmi, P. Rocca, M. Salucci, and A. Massa, "Optimisation of excitation tolerances for robust beamforming in linear arrays," IET Microw. Antennas Propag., vol. 10, no. 2, pp. 208-214, Jan. 2016.

[23] G. Gottardi, L. Poli, P. Rocca, A. Montanari, A. Aprile, and A. Massa, "Optimal monopulse beamforming for side-looking airborne radars," IEEE Antennas Wirel. Propag. Lett., vol. 16, pp. 1221-1224, Nov. 2016.

[24] G. Gottardi, N. Ebrahimi, P. Rocca, and A. Massa, "Optimal synthesis of monopulse beamforming weights for airborne radars through convex optimization," in Proc. Int. Applied Computational Electromagnetics Society Symp., Firenze, Italy, March 2017, pp. 1-2.

[25] P. Kasemir, N. Sutton, M. Radway, B. Jeong, T. Brown, and D. S. Filipovič, "Wideband analog and digital beamforming," in Proc. 2009 9th International Conference on Telecommunication in Modern Satellite, Cable, and Broadcasting Services, Nis, Serbia, Oct. 2009, pp. $372-375$.

[26] P. Mousavi, M. Fakharzadeh, and S. Safavi-Naeini, "1K element antenna system for mobile direct broadcasting satellite reception," IEEE Trans. Broadcast., vol. 56, no. 3, pp. 340-349, Sep. 2010.

[27] N. A. Sutton and D. S. Filipović, "V-band monolithically integrated four-arm spiral antenna and beamforming network," in Proc. 2012 IEEE International Symposium on Antennas and Propagation, Chicago, IL, USA, July 2012, pp. 1-2.

[28] L. Yang, G. Ren, W. Zhai, and Z. Qiu, "Beamforming based receiver scheme for DVB-T2 system in high speed train environment," IEEE Trans. Broadcasting, vol. 59, no. 1, pp. 146-154, Mar. 2013.

[29] A. Young, M. V. Ivashina, R. Maaskant, O. A. Iupikov, and D. B. Davidson, "Improving the calibration efficiency of an array fed reflector antenna through constrained beamforming," IEEE Trans. Antennas Propag., vol. 61, no. 7, pp. 3538-3545, July 2013. 
[30] D. S. Prinsloo, M. V. Ivashina, R. Maaskant, and P. Meyer, "Beamforming strategies for active multi-mode antennas: Maximum gain, signal-to-noise ratio, and polarization discrimination," in Proc. 2014 International Conference on Electromagnetics in Advanced Applications, Palm Beach, Aruba, Sep. 2014, pp. 507-510.

[31] O. Jo, W. Hong, S. T. Choi, S. Chang, C. Kweon, J. Oh, and K. Cheun, "Holistic design considerations for environmentally adaptive $60 \mathrm{GHz}$ beamforming technology," IEEE Commun. Mag., vol. 52, no. 11, pp. 30-38, Nov. 2014.

[32] O. A. Iupikov, M. V. Ivashina, K. Pontoppidan, P. H. Nielsen, C. Cappellin, N. Skou, S. S. Sobjaerg, A. Ihle, D. Hartmann, and K. V. 'T Klooster, "An optimal beamforming algorithm for phased-array antennas used in multi-beam spaceborne radiometers," in Proc. 2015 9th European Conference on Antennas and Propagation, Lisbon, Portugal, Aug. 2015, pp. 1-5.

[33] O. A. Iupikov, M. V. Ivashina, C. Cappellin, and N. Skou, "Digitalbeamforming array antenna technologies for future ocean-observing satellite missions," in Proc. IEEE Antennas and Propagation Society International Symposium, Fajardo, Puerto Rico, June-July 2016, pp. 1377-1378.

[34] O. A. Iupikov, M. V. Ivashina, N. Skou, C. Cappellin, K. Pontoppidan, and C. G. M. Van'T Klooster, "Multibeam focal plane arrays with digital beamforming for high precision space-borne ocean remote sensing," IEEE Trans. Antennas Propag., vol. 66, no. 2, pp. 737-748, Feb. 2018.

[35] C. A. Olen and R. T. Compton, "A numerical pattern synthesis algorithm for arrays," IEEE Trans. Antennas Propag., vol. 38, no. 10, pp. 1666-1676, Oct. 1990.

[36] H. Lebret and S. Boyd, "Antenna array pattern synthesis via convex optimization," IEEE Trans. Signal Process., vol. 45, no. 3, pp. 526-532, Mar. 1997.

[37] W. P. M. N. Keizer, "Fast low-sidelobe synthesis for large planar array antennas utilizing successive fast fourier transforms of the array factor," IEEE Trans. Antennas Propag., vol. 55, no. 3, pp. 715-722, Mar. 2007.

[38] R. C. Hansen, Phased array antennas. New Jersey: John Wiley \& Sons, Inc., 2009, pp. 49-107.

[39] G. K. Mahanti, V. V. B. Bikkani, and S. K. Mandal, "Placement of wide nulls in the radiation pattern of a linear array antenna using iterative Fast Fourier transform," in Proc. 2012 IEEE International Conference on Signal Processing, Communication and Computing, Hong Kong, China, 2012, pp. 552-555.

[40] F. E. Fakoukakis and G. A. Kyriacou, "Novel nolen matrix based beamforming networks for series-fed low SLL multibeam antennas," Prog. Electromagn. Res. B, vol. 51, pp. 33-64, 2013.

[41] R. Kumar, S. K. Mohanty, and B. B. Mangaraj, "Side lobe level and null control design of linear antenna array using Taguchi's method," in Proc. 2015 Intelligent Computing, Communication and Devices, Bhubaneswar, India, Apr. 2014, pp. 211-216.

[42] M. C. Johnson, S. L. Brunton, N. B. Kundtz and J. N. Kutz, "Sidelobe canceling for reconfigurable holographic metamaterial antenna," IEEE Trans. Antennas Propag., vol. 63, no. 4, pp. 1881-1886, Apr. 2015.

[43] Y. Liu, S. Chen, L. Zhang and Q. H. Liu, "Determining the first-null mainlobe region of an arbitrary pattern for 2-D numerical pattern synthesis algorithm," IEEE Trans. Antennas Propag., vol. 64, no. 3, pp. 1130-1136, Mar. 2016.

[44] Y. Yang, L. Yang, and J. Zhu, "Sidelobe suppression for time-domain adaptive broadband beamforming with sparse constraint," in Proc. OCEANS 2016 MTS/IEEE Monterey, Monterey, CA, USA, Sep. 2016, pp. $1-4$.

[45] M. A. Sarker, M. S. Hossain, and M. S. Masud, "Robust beamforming synthesis technique for low side lobe level using taylor excited antenna array," in Proc. 2016 2nd International Conference on Electrical, Computer \& Telecommunication Engineering, Rajshahi, Bangladesh, Dec. 2016, pp. 1-4.

[46] A. F. Morabito and P. G. Nicolaci, "Optimal synthesis of shaped beams through concentric ring isophoric sparse arrays," IEEE Antennas Wirel. Propag. Lett., vol. 16, pp. 979-982, 2017.

[47] L. Pulido-Mancera, M. F. Imani and D. R. Smith, "Discrete dipole approximation for simulation of unusually tapered leaky wave antennas," in Proc. 2017 IEEE MTT-S International Microwave Symposium, Honololu, HI, USA, June 2017, pp. 409-412.
[48] Y. Liu, X. Huang, K. D. Xu, Z. Song, S. Yang and Q. H. Liu, "Pattern synthesis of unequally spaced linear arrays including mutual coupling using iterative FFT via virtual active element pattern expansion," IEEE Trans. Antennas Propag., vol. 65, no. 8, pp. 3950-3958, Aug. 2017.

[49] S. E. El-Khamy, A. S. Eltrass, and H. F. El-Sayed, "Design of thinned fractal antenna arrays for adaptive beam forming and sidelobe reduction," IET Microw. Antennas Propag., vol. 12, no. 3, pp. 435-441, Feb. 2018. 